\title{
Dynamic In Vivo SPECT Imaging of Neural Stem Cells Functionalized with Radiolabeled Nanoparticles for Tracking of Glioblastoma
}

\author{
Shih-Hsun Cheng*1, Dou Yu*2, Hsiu-Ming Tsai ${ }^{1}$, Ramin A. Morshed ${ }^{2}$, Deepak Kanojia ${ }^{2}$, Leu-Wei Lo ${ }^{1,3}$, Lara Leoni ${ }^{1}$, \\ Yureve Govind $^{2}$, Lingjiao Zhang ${ }^{2}$, Karen S. Aboody ${ }^{4}$, Maciej S. Lesniak ${ }^{2}$, Chin-Tu Chen ${ }^{1}$, and Irina V. Balyasnikova ${ }^{2}$ \\ ${ }^{1}$ Department of Radiology, The University of Chicago, Chicago, Illinois; ${ }^{2}$ The Brain Tumor Center, The University of Chicago, \\ Chicago, Illinois; ${ }^{3}$ Institute of Biomedical Engineering and Nanomedicine, National Health Research Institute(s), Taiwan; and \\ ${ }^{4}$ Department of Neuroscience, City of Hope National Medical Center and Beckman Research Institute, Duarte, California
}

\begin{abstract}
There is strong clinical interest in using neural stem cells (NSCs) as carriers for targeted delivery of therapeutics to glioblastoma. Multimodal dynamic in vivo imaging of NSC behaviors in the brain is necessary for developing such tailored therapies; however, such technology is lacking. Here we report a novel strategy for mesoporous silica nanoparticle (MSN)-facilitated NSC tracking in the brain via SPECT. Methods: ${ }^{111}$ In was conjugated to MSNs, taking advantage of the large surface area of their unique porous feature. A series of nanomaterial characterization assays was performed to assess the modified MSN. Loading efficiency and viability of NSCs with ${ }^{111}$ In-MSN complex were optimized. Radiolabeled NSCs were administered to glioma-bearing mice via either intracranial or systemic injection. SPECT imaging and bioluminescence imaging were performed daily up to $48 \mathrm{~h}$ after NSC injection. Histology and immunocytochemistry were used to confirm the findings. Results: ${ }^{111}$ In-MSN complexes show minimal toxicity to NSCs and robust in vitro and in vivo stability. Phantom studies demonstrate feasibility of this platform for NSC imaging. Of significance, we discovered that decayed ${ }^{111} \mathrm{In}-\mathrm{MSN}$ complexes exhibit strong fluorescent profiles in preloaded NSCs, allowing for ex vivo validation of the in vivo data. In vivo, SPECT visualizes actively migrating NSCs toward glioma xenografts in real time after both intracranial and systemic administrations. This is in agreement with bioluminescence live imaging, confocal microscopy, and histology. Conclusion: These advancements warrant further development and integration of this technology with MRI for multimodal noninvasive tracking of therapeutic NSCs toward various brain malignancies.
\end{abstract}

Key Words: nanoparticle; neural stem cells; glioma; cell tracking; SPECT

J Nucl Med 2016; 57:279-284

DOI: 10.2967/jnumed.115.163006

Received Jun. 30, 2015; revision accepted Nov. 9, 2015.

For correspondence or reprints contact either of the following:

Irina V. Balyasnikova, Department of Neurological Surgery, Northwestern

University, 303 E. Superior St., Chicago, IL 60611.

E-mail: irinabal@northwestern.edu

Chin-Tu Chen, Department of Radiology, The University of Chicago, 5841

South Maryland Ave., MC2026, Chicago, IL 60637.

E-mail: cchen3@uchicago.edu

${ }^{*}$ Contributed equally to this work.

Published online Nov. 12, 2015.

COPYRIGHT (c) 2016 by the Society of Nuclear Medicine and Molecular Imaging, Inc.
$\mathbf{G}$

lioblastoma multiforme is the most aggressive primary malignant brain tumor in adults despite a maximal treatment regimen $(1,2)$. Lack of efficacious therapy along with the impermeable blood-brain barrier prompted the development of novel approaches for direct delivery of therapeutics to the tumor burden $(3,4)$. The nonimmunogenic nature and intrinsic ability of stem cells to preferentially migrate toward both local and disseminated diseases have generated much interest in their use as vehicles for targeted delivery of antitumor therapeutics (5-8). In particular, the success of preclinical studies investigating tropism, distribution, safety, and therapeutic efficacy of a clonal human neural stem cell (NSC) line, HB1.F3.CD, has led to approval of these cells as therapeutic carriers in patients with recurrent high-grade glioma (8). Thus, modalities tracking the fate of stem cells in vivo are of great interest and are critical for the tailored development of efficient stem cell-based glioma therapies.

SPECT, a nuclear imaging technique, possesses high sensitivity with minimal limit to tissue penetration (9) and can offer valuable information for interpretation of signal in the brain alone or in combination with MRI. The most widely used SPECT radionuclides include ${ }^{111} \mathrm{In}$ (half-life, $67 \mathrm{~h}$ ) and metastable ${ }^{99 \mathrm{~m}} \mathrm{Tc}$ (halflife, $6 \mathrm{~h}$ ). Although ${ }^{99 \mathrm{~m}} \mathrm{Tc}$ can be used in higher doses to improve the short-term imaging resolution, ${ }^{111}$ In can provide a longer time window for cell tracking. Indeed, several groups have used ${ }^{111}$ In to image mesenchymal stem cells (MSCs) in vivo around sites of myocardial injury in canine $(10,11)$ and porcine animal models (12). Human clinical studies have also used ${ }^{111}$ In-oxine $(13,14)$ and ${ }^{99 m}$ Tc-hexamethylpropleneamine oxine $(15-17)$ to assess stem cell trafficking in acute and chronic myocardial infarction. Nanoparticles are new modalities for labeling of stem cells and can offer advantages over other labeling methods (18). Mesoporous silica nanoparticles (MSNs) are particularly attractive as drug/contrast agent carriers because of their intrinsically large surface areas, accessible pores, low toxicity (19), and ease of uptake and transport by cells (20).

There is unmet need for development of imaging techniques for tracking of stem cells in the brain. Here we evaluate in vivo tracking of ${ }^{111} \mathrm{In}-\mathrm{MSN}$-labeled NSCs migrating toward glioma xenograft. This report validates that the ${ }^{111}$ In-MSN radiolabeling strategy enables SPECT imaging of in vivo behaviors of NSCs after either local or systemic delivery. 


\section{MATERIALS AND METHODS}

All reagents were used as received without further purification (Supplemental Table 1; supplemental materials are available at http:// jnm.snmjournals.org).

\section{Synthesis and Characterization of MSN}

To synthesize MSN, the sol-gel co-condensation of tetraethyl orthosilicate was performed (Supplemental Table 2). All samples were collected by centrifugation $(12,000 \mathrm{rpm} \times 20 \mathrm{~min})$ and a series of washes with deionized water and ethanol. Nanoparticles were characterized via transmission electron microscopy, dynamic light scattering, $\zeta$ potential, and nitrogen adsorption/desorption isotherm measurements to define topology, size, pore volume, surface area, and charge (supplemental materials).

\section{Radiolabeling of MSN with ${ }^{111}$ In and Loading of NSCs with ${ }^{111}$ In-MSN}

MSNs were conjugated with DOTA- $N$-hydroxysuccinimide-ester through amide formation (Fig. 1). The detailed optimization of labeling efficiency and stability assessments are described in the supplemental material. Inductively coupled plasma mass spectrometry was performed to quantify silicon and cadmium content in MSN (supplemental materials). Human NSCs (clone HB1.F3.CD; ClinicalTrials.gov identifier NCT01172964) were used in all in vitro and in vivo experiments. The uptake efficiency, cytotoxicity, and phantom study details are listed in the supplemental materials.

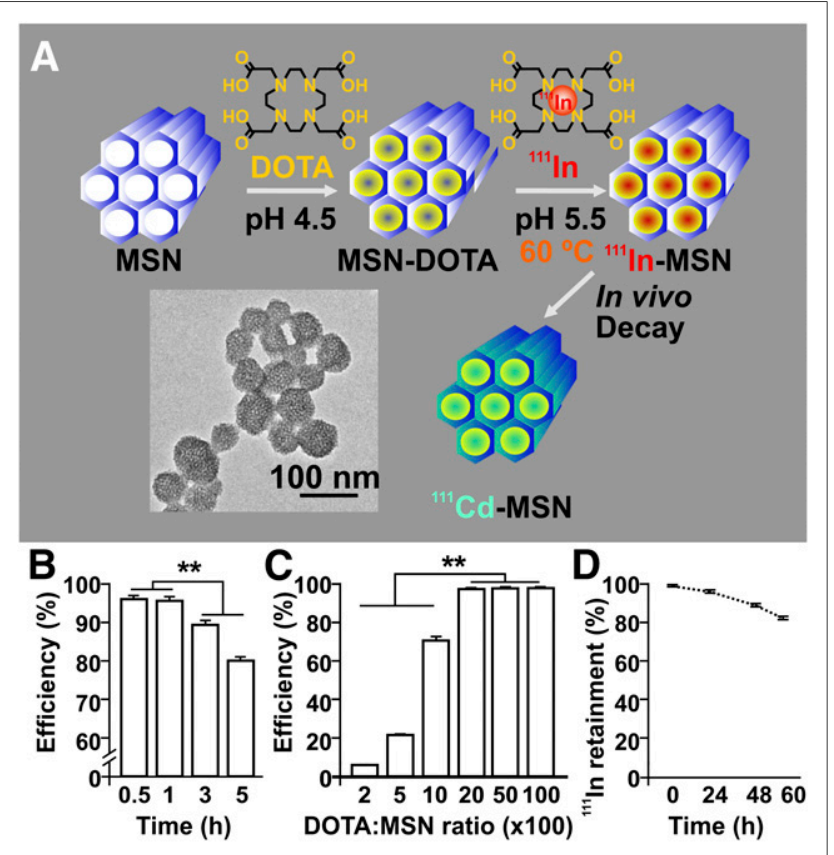

FIGURE 1. Optimization of loading protocol of MSN with ${ }^{111} \mathrm{In}$. (A) Schematic illustration of ${ }^{111}$ In encapsulation process in MSN. Uniformly sized MSNs were first modified with $\mathrm{NH}_{2}$ groups to form $\mathrm{MSN}-\mathrm{NH}_{2}$, followed by DOTA conjugation to yield MSN-DOTA via (2-(1H-benzotriazol1-yl)-1,1,3,3-tetramethyluronium hexafluorophosphate) (HBTU) coupling. ${ }^{111}$ In-MSN was radiolabeled in 2-( $\mathrm{N}$-morpholino)ethanesulfonic acid (MES) buffer at $60^{\circ} \mathrm{C}$. On decay, ${ }^{111} \mathrm{In}-\mathrm{MSN}$ transformed to ${ }^{111} \mathrm{Cd}-\mathrm{MSN}$ and acquired fluorescence spectra (Supplemental Fig. 2). Inset is transmission electron microscopy image of MSN-DOTA complexes. Scale bar $=100 \mathrm{~nm}$. (B) Time course of labeling efficiencies of ${ }^{111} \mathrm{In}-\mathrm{MSN}$ indicates on high efficiency within $0.5 \mathrm{~h}$. (C) Labeling efficiency of ${ }^{111} \mathrm{In}-\mathrm{MSN}$ with various DOTA:MSN molecule ratios indicates optimal ratio is greater than 2,000:1 ( $n=3, P<0.005$, paired $t$ test). (D) Stability of ${ }^{111}$ In retention by MSN sustained over $60 \mathrm{~h} .{ }^{\star \star} P<0.01$.

\section{Orthotopic Glioblastoma Model and ${ }^{111}$ In-MSN-NSC Injection}

The animal procedures were approved by the University of Chicago Institutional Animal Care and Use Committee. Male athymic mice (age, $6 \mathrm{wk}$ ) were intracranially injected with $1 \times 10^{5}$ U87MG cells in the right frontal lobe as previously described (21). Tumor cells were allowed to engraft for $3 \mathrm{wk}$ before SPECT imaging. Naïve, nontumorbearing animals served as controls. NSCs were prepared for in vivo imaging experiments as follows. NSCs were incubated with $150 \mu \mathrm{g} / \mathrm{mL}$ particles for $2 \mathrm{~h}$ in Opti-MEM medium (Invitrogen), subsequently washed twice, trypsinized, and collected by centrifugation in Dulbecco modified Eagle medium containing 10\% fetal bovine serum. After an additional washing, the cells were resuspended in sterile phosphatebuffered saline. Mice were anesthetized with ketamine/xelazine and inoculated with $1 \times 10^{6}$ of radiolabeled NSCs contralateral to the tumor injection site or with $3 \times 10^{6}$ cells through intracardiac systemic injection.

\section{SPECT/CT Imaging}

The mice were imaged via SPECT/CT and optical imaging at the indicated time points. Small-animal SPECT/CT imaging was performed at the University of Chicago Integrated Small Animal Imaging Research Resource on a Trifoil Triumph Trimodality preclinical microPET/SPECT/CT imaging system. CT imaging of animals was performed for anatomic coregistration, and CT fusion of SPECT images was performed using AMIRA software (FEI Co.). Acquisition strategy details are listed in the supplemental materials. Optical imaging of NSCs-fluc was performed using an IVIS Spectrum System with Living Image software (PerkinElmer) as previously described (21). For quantification of SPECT signal, the images were processed in the Image J software (National Institutes of Health). Regions of interest (33.75 mm) were drawn in 5 contiguous coronal slices in the left hemisphere and in the same region of the contralateral side of brain. The sum of the counts within the regions of interest was obtained by subtracting the background counts.

\section{Biodistribution of ${ }^{111}$ In-MSN Complex and Tissue Analysis}

Biodistribution studies after an intracranial injection of radiolabeled NSCs were performed $48 \mathrm{~h}$ after injection of ${ }^{111} \mathrm{In}$-MSN-loaded NSCs in the mouse brain. Details are listed in the supplemental materials and results in Supplemental Figure 4. On completion of SPECT imaging, the brains were flash-frozen and sectioned after ${ }^{111} \mathrm{In}$ decayed to the negligible level (details are provided in the supplemental materials).

\section{Statistical Analysis}

Statistical analyses were performed using Prism 4 (GraphPad Software Inc.). Sample size for each group was 3 or more, and numeric data were reported as mean $\pm \mathrm{SEM}$. Comparisons between 2 groups were conducted using the Student $t$ test, and differences between more than 2 groups were assessed using ANOVA with Tukey post hoc test or repeated-measures ANOVA with Bonferroni post hoc test. The Pearson coefficient was calculated for the correlation between tumor weight and accumulated radioactivity. All reported $P$ values were 2sided and considered to be statistically significant at $P<0.05, P<$ 0.01 , and $P<0.001$

\section{RESULTS}

\section{Radiochemistry of ${ }^{111}$ In-MSN Complex for SPECT Imaging}

A unique feature of MSN is the large surface area that supports efficient loading with radioisotopes or drug of choice. Previously, we demonstrated low toxicity of MSNs toward NSCs, confirming their feasibility for NSC-mediated antitumor therapies (20). Here, we designed radiolabeled MSN as a nanoscale probe for labeling and tracking NSCs within the brain toward glioma xenograft tissue 
using SPECT imaging. MSN synthesis was described previously (11), and the incorporation of ${ }^{111} \mathrm{In}$ is schematically shown in Figure 1A. MSNs were on average $70 \mathrm{~nm}$ in diameter with uniform morphology as seen with transmission electron microscopy. Supplemental Table 3 summarizes the physical properties of MSNs. Figure 1B shows that the labeling efficacy reaches maximum reaction in $30 \min (n=3)$. Up to $95 \%$ of ${ }^{111}$ In labeling efficacy was achieved at a molecular ratio (DOTA/MSN) of 2,000. (Fig. 1C; $n=3$ ). The average activity of MSN-DOTA- ${ }^{111}$ In was $21.2 \mathrm{MBq} / \mathrm{mg}$. Specifically, $0.5 \mathrm{mg} / \mathrm{mL}$ of MSN-DOTA- ${ }^{111} \mathrm{In}(n=3)$ were incubated in serum-free Dulbecco modified Eagle medium at $37^{\circ} \mathrm{C}$ and then analyzed for released radioactivity by instant thin-layer chromatography. The stability was more than $90 \%$ during the first $48 \mathrm{~h}$ and the release of ${ }^{111} \mathrm{In}$ reached only $18 \% \pm 2.3 \%$ after $60 \mathrm{~h}$ of incubation (Fig. 1D).

\section{NSC Uptake Efficacy and Toxicity Studies}

NSCs plated in 96 -well plates $(1.0 \times 105$ cell/well $)$ were treated with $100 \mu \mathrm{g} / \mathrm{mL}$ of ${ }^{111} \mathrm{In}-\mathrm{MSN}$ in Dulbecco modified Eagle medium containing $1 \%$ fetal bovine serum, reduced-serum OptiMEM medium, or in the presence of X-tremeGENE HP DNA transfection reagent (Roche Diagnostics Corporation) for 2 or 6 h. The uptake efficacy of ${ }^{111}$ In-MSN by NSCs was measured in a $\gamma$-counter. Figure $2 \mathrm{~A}$ shows that the uptake of ${ }^{111} \mathrm{In}-\mathrm{MSN}$ reached $58 \% \pm 1.7 \%$ when labeling was performed in Opti-MEM medium without significant improvement in the presence of transfection reagent and $33 \% \pm 0.9 \%$ in serum containing medium after 2 $\mathrm{h}$ of incubation. The 6-h treatment did not further improve the ${ }^{111}$ In-MSN uptake and was deemed unnecessary (data not shown). The presence of albumin and $\gamma$-globulins in serum containing medium is likely the main contributor to the observed differences

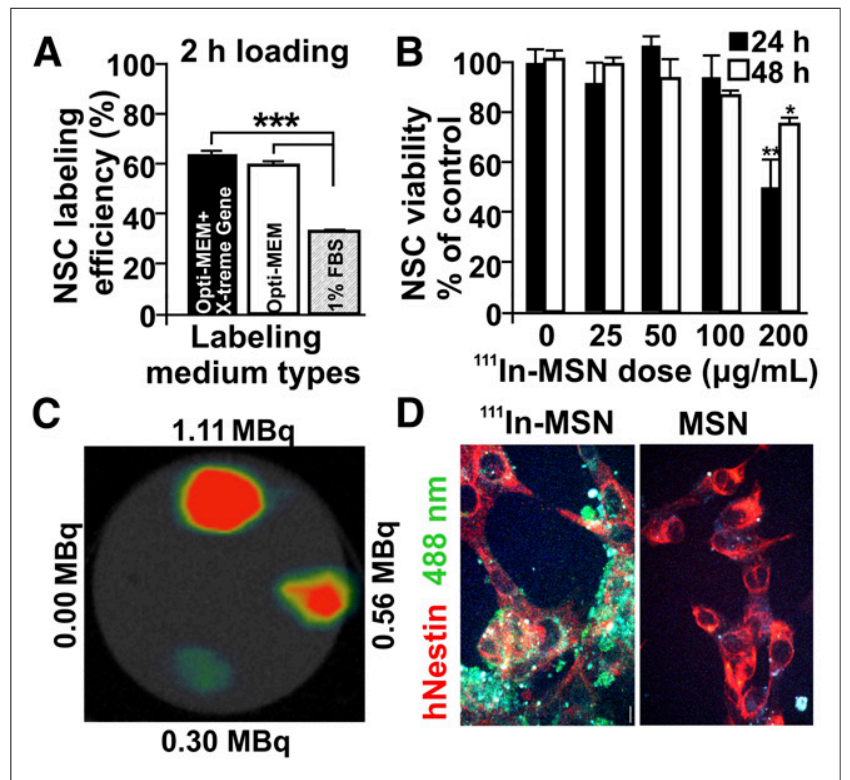

FIGURE 2. Loading of NSCs with ${ }^{111} \mathrm{In}-\mathrm{MSN}$ complex. (A) NSC culture medium conditions are a factor in loading efficiency of ${ }^{111} \mathrm{In}-\mathrm{MSN}$. (B) Viability study for safe loading in NSCs demonstrated preservation of NSC viability at doses $100 \mu \mathrm{g} / \mathrm{mL}$ or less in 24 and $48 \mathrm{~h}$. (C) Detection of ${ }^{111}$ In-MSN-loaded NSCs was validated via in vitro phantom study. (D) NSCs were visualized with phycoerythrin-conjugated antihuman nestin antibody (hNestin; red). Overlayed image of hNestin staining and fluorescence emitting decayed ${ }^{111} \mathrm{In}-\mathrm{MSN}$ excited at $488 \mathrm{~nm}$ (left). Nonradiolabeled MSNs in NSCs do not emit fluorescent signal (right). FBS = fetal bovine serum. in uptake $(22,23)$. In toxicity assays, cells were treated with 25 , 50, 100, and $200 \mu \mathrm{g} / \mathrm{mL}$ of ${ }^{111}$ In-MSN complex in Opti-MEM medium for $2 \mathrm{~h}$, after which the medium was changed to Dulbecco modified Eagle medium containing $10 \%$ fetal bovine serum for another 24 and 48 h. Figure $2 \mathrm{~B}$ shows that $94 \% \pm 8 \%(n=4)$ and $86 \% \pm 2 \%(n=4)$ of NSCs remained viable at $100 \mu \mathrm{g} / \mathrm{mL}$, whereby, viability was measured as $49 \% \pm 11 \%(n=3, P<$ $0.001)$ and $76 \% \pm 2 \%(n=4, P<0.05)$ of control at $200 \mu \mathrm{g}$ of ${ }^{111}$ In-MSN per milliliter at 24 and $48 \mathrm{~h}$, respectively. No toxicity of MSN to NSCs in the absence of ${ }^{111}$ In was detected (Supplemental Fig. 1). Thus, these studies identify the optimal protocol for loading NSCs with ${ }^{111} \mathrm{In}-\mathrm{MSN}$. Next, we performed an in vitro phantom study to validate SPECT feasibility. Figure $2 \mathrm{C}$ shows that SPECT signal is detectable in radiolabeled NSCs equal to $0.3 \mathrm{MBq}$ of ${ }^{111} \mathrm{In}$, corresponding to detectable levels in animal brains. Interestingly, we observed that fully decayed ${ }^{111}$ In-MSN emits fluorescence (Supplemental Fig. 2). Next, we characterized the fluorescent profiles of NSCs loaded with decayed ${ }^{111}$ In-MSN using confocal microscopy. Supplemental Fig. 2D shows confocal images of NSCs collected in a series of color channels. The green channel recorded the strongest fluorescent signal in NSCs loaded with decayed ${ }^{111} \mathrm{In}-\mathrm{MSN}$. Figure 2D shows that fluorescence of decayed ${ }^{111} \mathrm{In}-\mathrm{MSN}$ is strongly visible in overplayed images of NSCs stained with antinestin antibodies conjugated to phycoerythrin but not in NSCs loaded with nonradiolabeled MSN. Our data indicate that radiolabeled NSCs could be reliably traced in tissue sections based on the fluorescent properties of decayed ${ }^{111}$ In-MSN.

\section{In Vivo SPECT Imaging of NSCs in Mouse Glioma Xenograft Model After Intracerebral Injection}

For in vivo studies, athymic nude mice were implanted with a U87MG glioma cell line expressing cherry fluorescent protein in the right hemisphere of the brain. Three weeks later, control and tumorbearing animals were inoculated with NSCs-fluc (expressing firefly luciferase) loaded with radiolabeled MSN in the left hemisphere of the brain for subsequent SPECT/CT imaging. CT coregistration clearly shows the NSCs and glioma cell injection sites (Fig. 3A). The 3-dimensional views of SPECT images of control mice revealed that signal corresponding to radiolabeled NSCs is strictly localized at the injection site at day 0 , with no further distribution of the signal in days 1 and 2 after injection (Figs. 3B and 3C). Quantitative analysis of SPECT signal in 5 continuous slices of coronal view further confirmed this observation. In tumor-bearing animals, however, radiolabeled NSCs demonstrated robust migration as early as $4 \mathrm{~h}$ after injection $(47 \pm 1.3, P<0.001)$ which was sustained throughout day $1(34 \pm 2.1, P<0.001)$ and day $2(44 \pm 1.2, P<0.001)$ after injection (Figs. 3B and $3 \mathrm{C}$ ). Changes in the distribution at day 2 were highlighted by a more pronounced shift of the signal from the site of injection to the tumor site, with a definitive population of cells localized at the tumor implantation site. SPECT data corroborated bioluminescent imaging (BLI) of NSCs in control and tumorbearing animals (Supplemental Fig. 3)

\section{Histologic Evaluation of Distribution of ${ }^{111}$ In-MSN-Labeled NSCs in Brain After Intracerebral Injection}

Brain tissues were harvested and snap-frozen for histology analysis. Brain sections $(10 \mu \mathrm{m})$ were stained and scanned by the Pannoramic whole slide scanner (3DHistech Ltd.). Our discovery of decayed ${ }^{111}$ In-MSN-emitting fluorescence (peak at green spectrum, Supplemental Fig. 2) was used to identify radiolabeled NSCs in the brain tissue. Consistent with SPECT and BLI, histology 


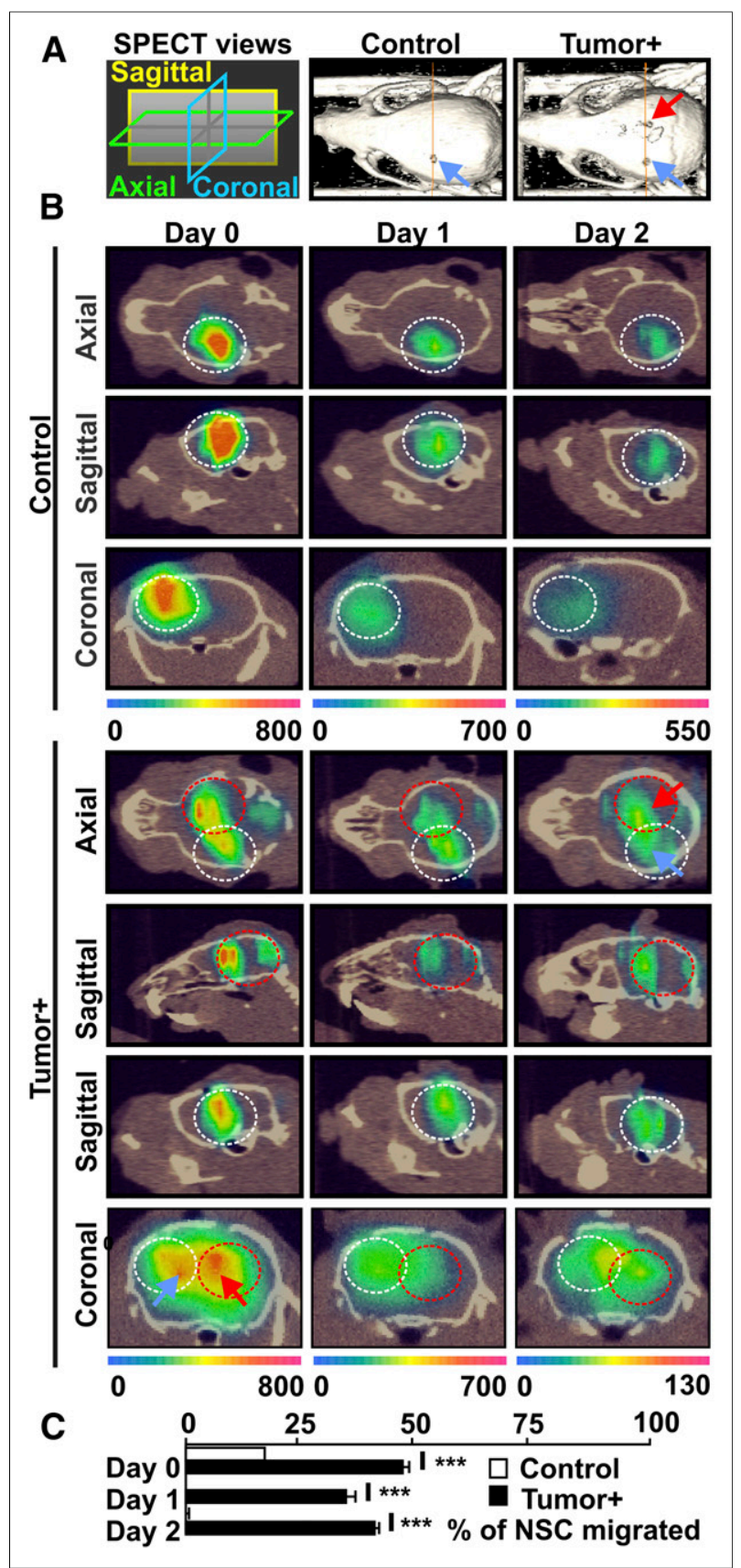

FIGURE 3. SPECT imaging of mouse brain after intracerebral delivery of NSCs loaded with ${ }^{111} \mathrm{In}-\mathrm{MSN}$. (A) Three-dimensional reconstruction of cranial CT scan shows NSC and glioma cell injection sites. (B) Radiolabeled NSCs were implanted in left cerebral hemisphere of control mouse $(n=3)$. SPECT scans show focalized signal corresponding to radiolabeled NSCs at injection site at days 0,1 , and 2. Imaging of radiolabeled NSCs in tumor-bearing mouse shows signal extending from injection site (white dashed circle) toward tumor-bearing site (red dashed circle) as early as $4 \mathrm{~h}$ after injection with further distribution of signal at days 1 and 2 and increased presence at tumor site. (C) SPECT signal was quantified in both hemispheres as described in "Materials and Methods" section. ${ }^{* \star} P<0.001$, repeated-measures ANOVA with Bonferroni post hoc test.

demonstrated confinement of NSCs at the injection site without distribution in control animals (data not shown). In contrast, Figure 4 shows mobile NSC populations corresponding to distrib- uted SPECT signals and, particularly, a population mobilized to the tumor site. Thus, with consistent evidence by immunocytochemistry, SPECT, and BLI, our data reliably distinguishes migrating NSCs in tumor-bearing animals $(7,21,24)$.

\section{Tracking ${ }^{111}$ In-MSN-NSCs in Mouse Brain After Systemic Injection}

NSCs have been shown to traverse the blood-brain barrier and migrate toward brain tumors (25). To further validate our tracking system, we explored the ability to visualize NSCs labeled with ${ }^{111}$ In-MSN in the mouse brain by SPECT after systemic injection. Figure 5A shows effective homing of NSCs toward the orthotopic xenograft site in the right cerebral hemisphere (cyan mass; yellow arrow; top), whereas the naïve control animal shows only nonspecific signal (coronal view of the fourth ventricle; bottom). Histology confirmed the growth of U87MG glioma in the experimental animal showing a localized SPECT signal and lack of such in naïve control animals (Fig. 5B). Confocal microscopy revealed partial intratumoral penetration of migrating NSCs with most positioned peritumorally, $48 \mathrm{~h}$ after the systemic delivery (Fig. 5B, top). The timeline of systemic SPECT imaging of NSC migration is consistent with their dynamics crossing the blood-brain barrier (25). In contrast, the control tissue showed only a sporadic nonmigratory signal (Fig. 5B, bottom). Thus, SPECT confirmed the ability of radiolabeled NSCs to penetrate the blood-brain barrier from circulation and localize in the tumor area (Supplemental Fig. 6).

\section{DISCUSSION}

Several in vivo stem cell tracking methods have been developed to understand their fate after transplantation. For example, iron

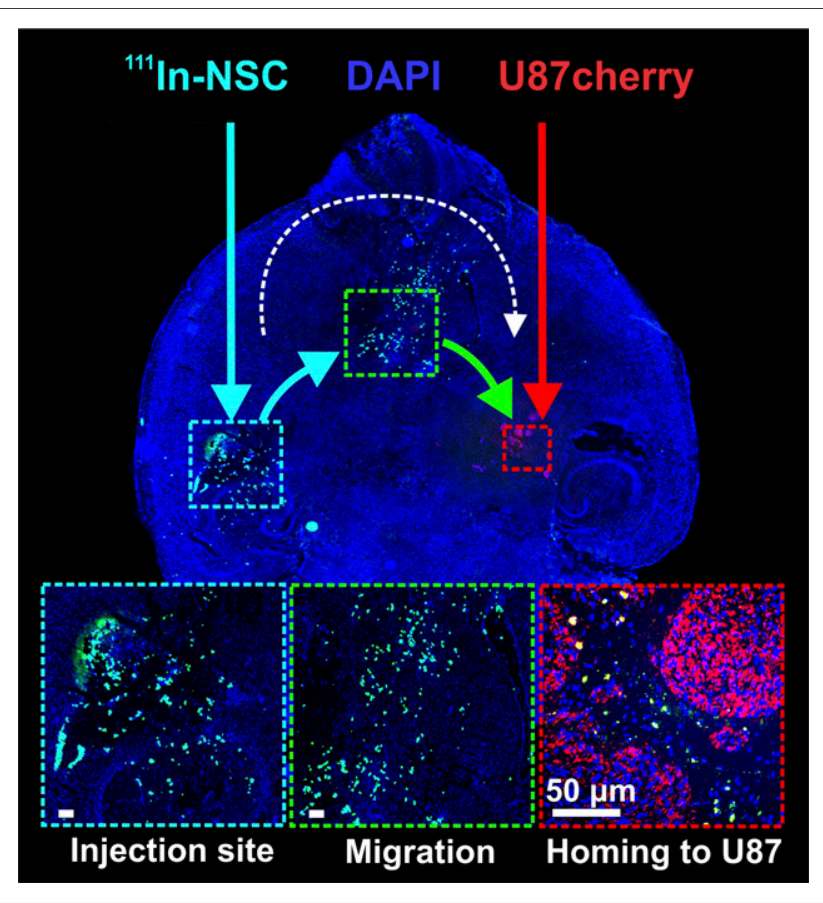

FIGURE 4. Confirmation of tumor tropism of NSCs loaded with ${ }^{111} \mathrm{In}-$ MSN in mouse brain by fluorescent microscopy. Whole-brain scan of tissue sections from tumor-bearing animal injected with NSCs loaded with ${ }^{111}$ In-MSN (green/cyan cells). NSCs can be seen at injection site, along migration path, and near U87cherry glioma xenograft. DAPI $=4$ ',6-diamidino2-phenylindole. 


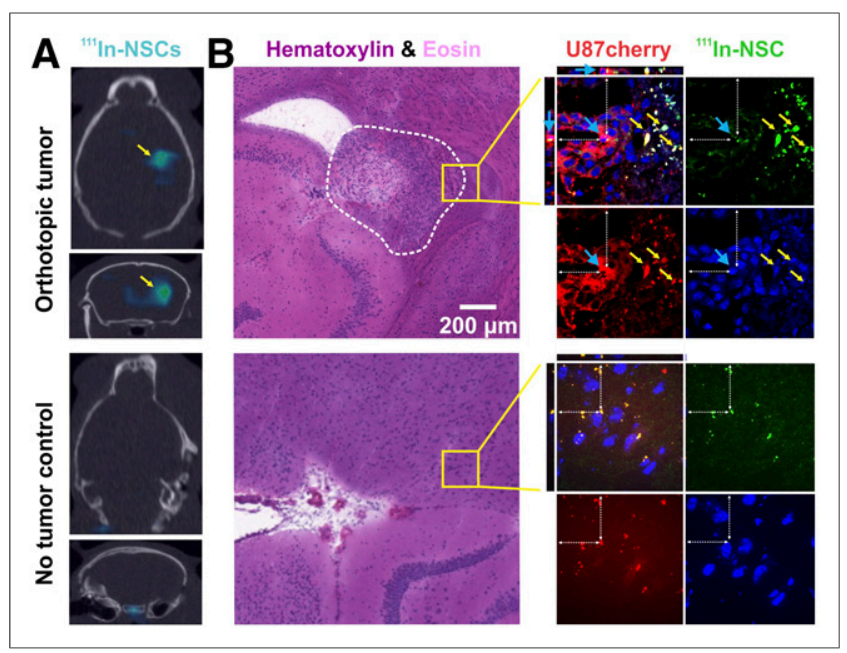

FIGURE 5. Tracking of NSCs in mouse brain after systemic injection. (A) SPECT imaging of ${ }^{111}$ In-MSN-NSCs after systemic injection in tumor-bearing (top) and control (bottom) animals. (B) Hematoxylin and eosin staining confirms orthotopic tumor growth (dashed white line) in experimental animals and normal structure of brain tissue in naïve control (lower). Confocal microscopy (yellow boxes) of adjacent tissue areas reveal strong homing behaviors of NSCs labeled with ${ }^{111}$ In-MSN (green; yellow arrows) near border of tumor mass (red), with occasional indications of intratumoral penetration by radiolabeled NSCs (blue arrow; orthogonal views). In contrast, naïve control tissue shows only sporadic residual SPECT signal of nonmigratory NSCs.

oxide nanoparticles have been used for tracking stem cells via MRI $(11,26)$. PET and SPECT $(11,27,28)$ have also applied to the experimental and clinical protocols for in vivo stem cell detection and their engraftment efficiency in the myocardium and other organs (29). Although bioluminescence and fluorescence optical imaging techniques have been successfully used in experimental animal models, their clinical relevance is lacking $(30,31)$. MRI has significant limitations due to false-positive signals arising from macrophage engulfment of imaging vehicles, small hemorrhages, or iron depositions in the aging or degenerating brain $(14,18,32,33)$. These limitations highlight the unmet need for additional in vivo stem cell tracking imaging modalities. Recent preliminary efforts in developing stem cell tracking strategies using nanoparticles and PET/MRI demonstrated the feasibility (34), but it requires further optimization and validation.

Our report uses SPECT for the in vivo tracking of migrating NSCs toward glioblastoma. Incorporation of PET or MRI coregistration of tumor along with SPECT could potentially enhance our knowledge of the behavior of stem cells in the brain. In addition, improvements in the loading capacity of SPECT isotopes in stem cells, the detection sensitivity, and the spatial resolution would also dramatically enhance the ability for in vivo stem cell tracking. The sensitivity and spatial resolution of the system could be significantly enhanced using advanced imaging equipment. Cai et al. reported the development of an ultrahigh-resolution MR-compatible SPECT system capable of providing a sub-500- $\mu \mathrm{m}$ imaging resolution (35). Meng et al. demonstrated that a dual-head camera single photon emission experimental system allowed the imaging of less than a thousand $\mathrm{T}$ cells inoculated in the brain corresponding to only $444 \mathrm{~Bq}$ of ${ }^{125} \mathrm{I}$ activity (36). This system can be readily translated into a full SPECT system for tracking of a small number of cells in vivo (37). We anticipate that the amount of ${ }^{111}$ In-MSN suitable for labeling of NSCs and established in our study will be sufficient for tracking of NSCs in clinical applications. In current clinical trials (NCT01172964; NCT02015819), patients with glioblastoma multiforme receive 50-100 times more NSCs in the resection cavity than intracranially injected in mice. It is, therefore, expected that the amount of radioactivity presented by NSCs $(\sim 101.75 \mathrm{MBq}$ of ${ }^{111}$ In corresponding to $5 \times 10^{7} \mathrm{NSCs}$ ) will be sufficient to generate reliable SPECT signals. In fact, Gholamrezanezhad et al. demonstrated that ${ }^{111}$ In-oxine-labeled MSCs injected in the patients (74266.4 MBq of ${ }^{111}$ In-oxine activity corresponding to $400 \times 10^{6}$ of systemically injected MSCs) could be imaged in multiple organs over days and were safe in patients with liver cirrhosis (38). The range of radioactivity sufficient for the tracking of NSCs within the human brain will be determined in future studies.

\section{CONCLUSION}

Our data demonstrate that NSCs labeled with ${ }^{111}$ In-MSN can be tracked as they migrate toward intracranial glioma xenografts by SPECT imaging; this imaging modality allowed the detection of NSC distribution in the brain after intracranial and systemic injections. Collective SPECT data corroborate BLI and confocal microscopy. Finally, our study suggests that this noninvasive in vivo imaging platform could help generate new knowledge of therapeutic stem cell behaviors, thus further advancing them into clinical practice.

\section{DISCLOSURE}

The costs of publication of this article were defrayed in part by the payment of page charges. Therefore, and solely to indicate this fact, this article is hereby marked "advertisement" in accordance with 18 USC section 1734 . This work was supported by funding from the NIH/National Institute of Neurological Disorders and Stroke (RO1 NS087990), the NIH/National Institute for Biomedical Imaging and Bioengineering (RO1 EB011640), and in part by funding provided by the Virginia and D.K. Ludwig Fund for Cancer Research via the Imaging Research Institute in the Biological Sciences Division, by the University of Chicago Comprehensive Cancer Center, and by the Department of Radiology at the University of Chicago. This project was also supported in part by the National Center for Advancing Translational Sciences of the National Institutes of Health through grant UL1 TR000430. The research of nanomaterials design and synthesis reported in this publication was also partially supported by BN-103-PP-04 and NM-103-PP-01 from the National Health Research Institutes of Taiwan and NSC102- 2113-M-400-001-MY3 from the National Science Council of Taiwan. Metal analysis was performed at the Northwestern University Quantitative Bioelemental Imaging Center generously supported by NASA Ames Research Center grant NNA04CC36G. Confocal imaging was performed at the University of Chicago Integrated Light Microscopy Facility. Karen S. Aboody is an uncompensated Board Member, Chief Scientific Officer, and shareholder of TheraBiologics. No other potential conflict of interest relevant to this article was reported.

\section{REFERENCES}

1. Stupp R, Mason WP, van den Bent MJ, et al. Radiotherapy plus concomitant and adjuvant temozolomide for glioblastoma. N Engl J Med. 2005;352:987-996.

2. Stupp R, Hegi ME, Mason WP, et al. Effects of radiotherapy with concomitant and adjuvant temozolomide versus radiotherapy alone on survival in glioblastoma in a randomised phase III study: 5-year analysis of the EORTC-NCIC trial. Lancet Oncol. 2009;10:459-466. 
3. Lesniak MS, Brem H. Targeted therapy for brain tumours. Nat Rev Drug Discov. 2004;3:499-508.

4. Sathornsumetee S, Rich JN. New approaches to primary brain tumor treatment. Anticancer Drugs. 2006;17:1003-1016.

5. Nakamizo A, Marini F, Amano T, et al. Human bone marrow-derived mesenchymal stem cells in the treatment of gliomas. Cancer Res. 2005;65:3307-3318.

6. Lee DH, Ahn Y, Kim SU, et al. Targeting rat brainstem glioma using human neural stem cells and human mesenchymal stem cells. Clin Cancer Res. 2009;15: 4925-4934.

7. Ahmed AU, Thaci B, Tobias AL, et al. A preclinical evaluation of neural stem cell-based cell carrier for targeted antiglioma oncolytic virotherapy. J Natl Cancer Inst. 2013;105:968-977.

8. Aboody KS, Najbauer J, Metz MZ, et al. Neural stem cell-mediated enzyme/ prodrug therapy for glioma: preclinical studies. Sci Transl Med. 2013;5:184ra59.

9. Naumova AV, Modo M, Moore A, Murry CE, Frank JA. Clinical imaging in regenerative medicine. Nat Biotechnol. 2014;32:804-818.

10. Blackwood KJ, Lewden B, Wells RG, et al. In vivo SPECT quantification of transplanted cell survival after engraftment using ${ }^{111}$ In-tropolone in infarcted canine myocardium. J Nucl Med. 2009;50:927-935.

11. Kraitchman DL, Tatsumi M, Gilson WD, et al. Dynamic imaging of allogeneic mesenchymal stem cells trafficking to myocardial infarction. Circulation. 2005; 112:1451-1461.

12. Lyngbaek S, Ripa RS, Haack-Sorensen M, et al. Serial in vivo imaging of the porcine heart after percutaneous, intramyocardially injected ${ }^{111}$ In-labeled human mesenchymal stromal cells. Int J Cardiovasc Imaging. 2010;26:273-284.

13. Caveliers V, De Keulenaer G, Everaert H, et al. In vivo visualization of ${ }^{111} \mathrm{In}$ labeled CD133+ peripheral blood stem cells after intracoronary administration in patients with chronic ischemic heart disease. $Q \mathrm{~J}$ Nucl Med Mol Imaging. 2007;51:61-66.

14. Kurpisz M, Czepczynski R, Grygielska B, et al. Bone marrow stem cell imaging after intracoronary administration. Int J Cardiol. 2007;121:194-195.

15. Goussetis E, Manginas A, Koutelou M, et al. Intracoronary infusion of CD133+ and CD133-CD34+ selected autologous bone marrow progenitor cells in patients with chronic ischemic cardiomyopathy: cell isolation, adherence to the infarcted area, and body distribution. Stem Cells. 2006;24:2279-2283.

16. Mesquita CT, Correa PL, Felix RC, et al. Autologous bone marrow mononuclear cells labeled with Tc-99m hexamethylpropylene amine oxime scintigraphy after intracoronary stem cell therapy in acute myocardial infarction. $\mathrm{J} \mathrm{Nucl} \mathrm{Cardiol}$. 2005;12:610-612.

17. Penicka M, Lang O, Widimsky $\mathrm{P}$, et al. One-day kinetics of myocardial engraftment after intracoronary injection of bone marrow mononuclear cells in patients with acute and chronic myocardial infarction. Heart. 2007;93:837-841.

18. Gu E, Chen WY, Gu J, Burridge P, Wu JC. Molecular imaging of stem cells: tracking survival, biodistribution, tumorigenicity, and immunogenicity. Theranostics. 2012;2:335-345.

19. Lee $\mathrm{CH}$, Cheng $\mathrm{SH}$, Wang YJ, et al. Near-infrared mesoporous silica nanoparticles for optical imaging: characterization and in vivo biodistribution. Adv Funct Mater. 2009; 19:215-222.

20. Cheng Y, Morshed R, Cheng SH, et al. Nanoparticle-programmed self-destructive neural stem cells for glioblastoma targeting and therapy. Small. 2013;9:4123-4129.

21. Balyasnikova IV, Prasol MS, Ferguson SD, et al. Intranasal delivery of mesenchymal stem cells significantly extends survival of irradiated mice with experimental brain tumors. Mol Ther. 2014;22:140-148.
22. Drescher D, Orts-Gil G, Laube G, et al. Toxicity of amorphous silica nanoparticles on eukaryotic cell model is determined by particle agglomeration and serum protein adsorption effects. Anal Bioanal Chem. 2011;400:1367-1373.

23. Stayton I, Winiarz J, Shannon K, Ma Y. Study of uptake and loss of silica nanoparticles in living human lung epithelial cells at single cell level. Anal Bioanal Chem. 2009;394:1595-1608.

24. Ahmed AU, Thaci B, Alexiades NG, et al. Neural stem cell-based cell carriers enhance therapeutic efficacy of an oncolytic adenovirus in an orthotopic mouse model of human glioblastoma. Mol Ther. 2011;19:1714-1726.

25. Gutova M, Frank JA, D'Apuzzo M, et al. Magnetic resonance imaging tracking of ferumoxytol-labeled human neural stem cells: studies leading to clinical use. Stem Cells Transl Med. 2013;2:766-775.

26. Edmundson M, Thanh NT, Song B. Nanoparticles based stem cell tracking in regenerative medicine. Theranostics. 2013;3:573-582.

27. Zhang Y, Ruel M, Beanlands RS, deKemp RA, Suuronen EJ, DaSilva JN. Tracking stem cell therapy in the myocardium: applications of positron emission tomography. Curr Pharm Des. 2008;14:3835-3853.

28. Chin BB, Nakamoto Y, Bulte JW, Pittenger MF, Wahl R, Kraitchman DL. ${ }^{111}$ In oxine labelled mesenchymal stem cell SPECT after intravenous administration in myocardial infarction. Nucl Med Commun. 2003;24:1149-1154.

29. McColgan P, Sharma P, Bentley P. Stem cell tracking in human trials: a metaregression. Stem Cell Rev. 2011;7:1031-1040.

30. Mirpour S, Gholamrezanezhad A. Clinical stem cell imaging and in vivo tracking. In: Gholamrezanezhad A, ed. Stem Cells in Clinic and Research. Rijeka, Croatia: InTech; 2011. InTech website. http://www.intechopen.com/ books/stem-cells-in-clinic-and-research/clinical-stem-cell-imaging-and-in-vivotracking. Accessed December 1, 2015.

31. Polzer H, Haasters F, Prall WC, et al. Quantification of fluorescence intensity of labeled human mesenchymal stem cells and cell counting of unlabeled cells in phase-contrast imaging: an open-source-based algorithm. Tissue Eng Part C Methods. 2010;16:1277-1285.

32. Pawelczyk E, Arbab AS, Chaudhry A, Balakumaran A, Robey PG, Frank JA. In vitro model of bromodeoxyuridine or iron oxide nanoparticle uptake by activated macrophages from labeled stem cells: implications for cellular therapy. Stem Cells. 2008;26:1366-1375.

33. Brass SD, Chen NK, Mulkern RV, Bakshi R. Magnetic resonance imaging of iron deposition in neurological disorders. Top Magn Reson Imaging. 2006;17:31-40.

34. Huang $X$, Zhang F, Wang $H$, et al. Mesenchymal stem cell-based cell engineering with multifunctional mesoporous silica nanoparticles for tumor delivery. Biomaterials. 2013;34:1772-1780.

35. Cai L, Lai X, Shen Z, Chen CT, Meng LJ. MRC-SPECT: a sub-500 $\mu$ m resolution MR-compatible SPECT system for simultaneous dual-modality study of small animals. Nucl Instrum Methods Phys Res A. 2014;734, Part B:147-151.

36. Meng LJ, Fu G, Roy EJ, Suppe B, Chen CT. An ultrahigh resolution SPECT system for I-125 mouse brain imaging studies. Nucl Instrum Methods Phys Res A. 2009;600:498-505.

37. Meng LJ, Fu G, Tan JW, Chen CT. Imaging performance of an sub-hundred micron resolution SPECT/CT system. Paper presented at: Institutes of Electrical and Electronic Engineers (IEEE) Nuclear Science Symposium and Medical Imaging Conference (NSS/MIC); October 28-November 3, 2007; Honolulu, Hawaii.

38. Gholamrezanezhad A, Mirpour S, Bagheri M, et al. In vivo tracking of ${ }^{111} \mathrm{In}$ oxine labeled mesenchymal stem cells following infusion in patients with advanced cirrhosis. Nucl Med Biol. 2011;38:961-967. 